\title{
Aspects of Upper Defensive Alliances
}

\author{
Cristina Bazgan ${ }^{1}{ }^{*} \quad$ Henning Fernau ${ }^{2} \quad$ Zsolt Tuza $^{3,4}{ }^{\dagger}$
}

1. Université Paris-Dauphine, PSL Research University, CNRS, LAMSADE, 75016 Paris, France bazgan@lamsade.dauphine.fr

2. Universität Trier, Fachbereich 4, Informatikwissenschaften

fernau@uni-trier.de

3. Alfréd Rényi Institute of Mathematics, Hungarian Academy of Sciences, Budapest, Hungary

4. Department of Computer Science and Systems Technology, University of Pannonia,

Veszprém, Hungary

tuza@dcs.uni-pannon.hu

\begin{abstract}
A defensive alliance in a graph $G=(V, E)$ is a set of vertices $S$ satisfying the condition that every vertex $v \in S$ has at least as many neighbors (including itself) in $S$ than it has in $V \backslash S$. We also consider strong defensive alliances where the vertex itself is not considered in the inequality. We consider two notions of minimality in this paper, local and global minimality and we are interested in minimal (strong) defensive alliances of maximum size. We also look at connected versions of these alliances. We show that these problems are NP-hard.
\end{abstract}

\section{Introduction}

Alliances in graphs were introduced first in 2000 by Kristiansen et al. in [13] and further studied by Shafique [17] and other authors. The purpose is to form coalitions of vertices able to defend each other from attacks of other vertices (in the case of defensive alliances) or able to collaborate to attack non-allied vertices (in the case of offensive alliances). Alliances can be formed between nations in a security context, between companies in a business context, or between people wishing to gather by affinity. Alliances can be viewed as communities. Identifying communities within social or biological networks, or within

\footnotetext{
${ }^{*}$ Institut Universitaire de France

${ }^{\dagger}$ Research supported in part by the National Research, Development and Innovation Office - NKFIH under the grant SNN 116095.
} 
the web graph, is a major and fashionable concern. In the web context, a community is defined by Flake et al. [7] as a set of web pages that links to more web pages in the community than to pages out of the community.

Various types of alliances were formally defined. In this study, we focus on defensive alliances. A defensive alliance is a set of vertices with the property that each vertex has at least as many neighbors in the alliance (counting itself in) as neighbors outside the alliance. A defensive alliance is strong if each vertex has at least as many neighbors in the alliance (not counting itself in) as outside the alliance. This last concept was defined by Kristiansen et al. in [13] and it corresponds to a satisfactory subset defined in [2,3]. More general, a $k$-defensive alliance is a vertex subset such that each vertex has at least $k$ more neighbors in the alliance than outside the alliance, see [15].

The theory of alliances in graphs was developed over the last decade both from a combinatorial and from a computational perspective. However, the focus has mostly been on finding small alliances, although studying large alliances do not only makes a lot of sense from the original motivation of these notions, but was actually also delineated in the very first papers on alliances. Carvajal et al. [4] proved that deciding if a graph contains a strong defensive alliance of size at most $\ell$ is NP-hard. This result was generalized to $k$-defensive alliances, for any $k \in\{-\Delta, \ldots, \Delta\}$ [18], where $\Delta$ is the maximum degree of the considered graph, and in particular for $k=-1$, the special case that corresponds to a defensive alliance. A survey establishing the main known results on defensive alliances in graphs can be found in [20].

Note that being a (strong) defensive alliance is not an hereditary property, that is, a set contained in a (strong) defensive alliance is not necessarily a (strong) defensive alliance. Shafique [17] called an alliance a locally minimal alliance if the set obtained by removing any vertex of the alliance is not an alliance. ${ }^{1}$ We also consider another notion of minimal alliance (called critical alliance or minimal alliance in [17]) that we call a globally minimal alliance or shorter minimal alliance which has the property that no proper subset is an alliance.

In this paper we are interested in (locally) minimal (strong) alliances of maximum size. Considering such notions can be well motivated by the community detection scenario mentioned above: clearly, big communities where every member still matters somehow are of more interest than really small communities. Also, there is a general mathematical interest in such type of problems, see [14].

The paper is organized as follows. Basic definitions and properties are given in Section 2. ${ }^{2}$ We also present examples that show that the graph parameters that we study are substantially different. Section 3 establishes complexity results of these problems. In particular, we prove NP-hardness results for all the graph parameters that we introduce in this paper, even on degree-bounded graphs. We finish with presenting some research directions.

\footnotetext{
${ }^{1}$ This corresponds to the notion of 1-minimality in [9].

${ }^{2}$ We assume knowledge on some basic notions of complexity theory, but we will indicate some related facts through this paper.
} 


\section{Basic notions and preliminary results}

Let us recall some basic notions. Let $G=(V, E)$ be a graph. We only consider simple undirected graphs, i.e., the edge relation $E \subseteq V \times V$ is assumed to be symmetric and irreflexive. The components of the smallest equivalence relation containing $E$ are known as connected components. A graph is connected if it has exactly one connected component. The open neighborhood of a vertex $v \in V$ is the set $N_{G}(v)=\{u \in V: u v \in E\}$ (or shortly $N(v)$ if $G$ is clear from the context), and the closed neighborhood of $v$ is the set $N_{G}[v]=N(v) \cup\{v\}$ (or shortly $N[v]$ ). The degree of $v$ is $d(v)=|N(v)|$ and the average degree of $G$ is equal to $2|E| /|V|$. A graph is called $k$-regular if all its vertices have degree $k$. A graph is called cubic if it is 3-regular. If $U \subseteq V$, then $G[U]$ denotes the graph induced by $U$, i.e., $G[U]=\left(U, E_{U \times U}\right)$, where $E_{U \times U}$ is the restriction of relation $E$ to the set $U$. We also write $d_{U}(v)=\left|N_{G[U \cup\{v\}]}(v)\right|$. If $I \subseteq V$ satisfies that $G[I]$ is 0-regular, then $I$ is called an independent set. A graph is bipartite if its vertex set can be partitioned into two independent sets. A cycle is a connected 2-regular graph. Removing exactly one edge from a cycle yields a path. $G^{\prime}=\left(V^{\prime}, E^{\prime}\right)$ is a subgraph of $G=(V, E)$ if $V^{\prime} \subseteq V$ and $E^{\prime} \subseteq E$; and $G^{\prime}$ is an induced subgraph if $G^{\prime}=G\left[V^{\prime}\right]$. An induced subgraph that forms a cycle is also known as a chordless cycle. A graph that can be embedded into the plane is called a planar graph. A graph $G=(V, E)$ is Hamiltonian if it has a subgraph $G^{\prime}=\left(V^{\prime}, E^{\prime}\right)$, with $V^{\prime}=V$, that is a cycle. A set $M$ of edges of $G=(V, E)$ is a matching if no two edges from $M$ share an endpoint.

A non-empty set $D \subseteq V$ is called

- a defensive alliance if $\forall v \in D:|N[v] \cap D| \geq|N(v) \backslash D|$;

- a strong defensive alliance if $\forall v \in D:|N(v) \cap D| \geq|N(v) \backslash D|$.

A (strong) defensive alliance is connected if the subgraph induced by $D$ is connected.

An alliance $D$ is called a locally minimal alliance if for any $v \in D, D \backslash\{v\}$ is not an alliance. An alliance is globally minimal alliance or shorter minimal alliance if no proper subset is an alliance. An alliance $D$ is called a connected locally minimal alliance if for any $v \in D, D \backslash\{v\}$ is not a connected alliance. Notice that any globally minimal alliance is also connected.

In this paper we use the following notations, introduced in [17] for global minimality. Hence, we use

- $A(G)$ for the cardinality of the largest minimal defensive alliance in a graph $G$, known as the upper defensive alliance number;

- $\hat{A}(G)$ for the cardinality of the largest minimal strong defensive alliance in a graph $G$, known as the upper strong defensive alliance number;

- $A_{L}(G)$ for the cardinality of the largest locally minimal defensive alliance in a graph $G$, called local upper defensive alliance number; 
- $\hat{A}_{L}(G)$ for the cardinality of the largest locally minimal strong defensive alliance in a graph $G$, called local upper strong defensive alliance number;

- $A_{c L}(G)$ for the cardinality of the largest connected locally minimal defensive alliance in a graph $G$, called connected local upper defensive alliance number and

- $\hat{A}_{c L}(G)$ for the cardinality of the largest connected locally minimal strong defensive alliance in a graph $G$, called connected local upper strong defensive alliance number.

Since any minimal (strong) defensive alliance is connected and it is a (strong) locally minimal defensive alliance we have $A(G) \leq A_{L}(G), A(G) \leq A_{c L}(G)$ and $\hat{A}(G) \leq \hat{A}_{L}(G)$, $\hat{A}(G) \leq \hat{A}_{c L}(G)$. However, $A_{c L}(G)$ (resp. $\hat{A}_{c L}(G)$ ) could be smaller or larger than $A_{L}(G)$ (resp. $\left.\hat{A}_{L}(G)\right)$.

Some upper bounds for the upper defensive alliance numbers are also contained in [13].

Example 1 We present a family of graphs where $A_{c L}$ (and $\hat{A}_{c L}$, respectively) is arbitrarily smaller than $A_{L}$ (and $\hat{A}_{L}$, respectively).

In a cycle $C_{n}=\left\{v_{1}, \ldots, v_{n}\right\}$ of size $n=3 t, A\left(C_{n}\right)=\hat{A}\left(C_{n}\right)=A_{c L}\left(C_{n}\right)=\hat{A}_{c L}\left(C_{n}\right)=2$ (namely, take any two adjacent vertices, for example $\left.\left\{v_{1}, v_{2}\right\}\right), A_{L}\left(C_{n}\right)=\hat{A}_{L}\left(C_{n}\right)=2 n / 3$ (take all vertices except $v_{i}$ with $i=1 \bmod 3$, that is, an induced maximum matching). In order to see that $\hat{A}\left(C_{n}\right)=2$, observe that $C_{n}$ is not a connected locally minimal defensive alliance, and a path is a connected locally minimal defensive alliance if and only if it is an edge, otherwise an end-vertex can be removed.

Example 2 We exhibit a family of graphs where $\hat{A}_{c L}$ is arbitrarily larger than $\hat{A}_{L}$.

Consider the graph $G_{n}$ on $n$ vertices from Figure 1 where all vertices are of degree 2 except the 10 vertices from the gadgets at the left and at the right, that are of degree 3. We have $\hat{A}\left(G_{n}\right)=4$ (namely, consider a chordless cycle of size 4 , for example $\left\{a_{2}, a_{3}, a_{5}, a_{4}\right\}$ ), $\hat{A}_{c L}\left(G_{n}\right)=n-2$ (take all vertices except $a_{1}$ and its symmetric counterpart in the left gadget), $\hat{A}_{L}\left(G_{n}\right) \approx 2 n / 3$ (this is seen by considering a cycle of size 3, for example $a_{1}, a_{2}, a_{3}$, followed on the path by pairs of consecutive vertices $\left\{a_{6}, a_{7}\right\},\left\{a_{9}, a_{10}\right\}, \ldots$, skipping every third vertex).

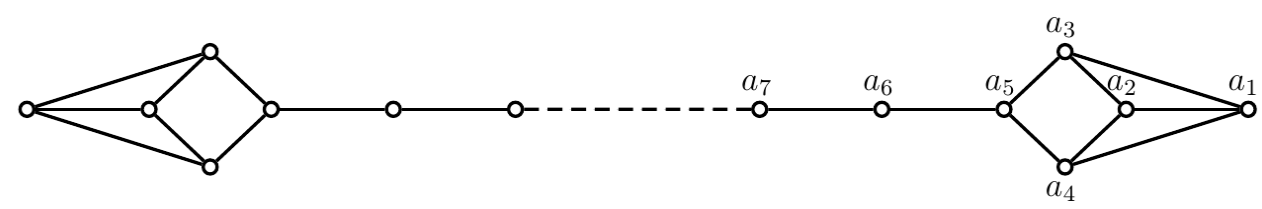

Figure 1: A graph $G$ with $\hat{A}\left(G_{n}\right)<\hat{A}_{L}\left(G_{n}\right)<\hat{A}_{c L}\left(G_{n}\right)$

Example 3 We show a family of graphs where $A_{c L}$ is arbitrarily larger than $A_{L}$.

Consider the graph $G_{n}^{\prime}$ on $n$ vertices from Figure 2 where all vertices are of degree 2 except the 12 vertices from the gadgets at the left and at the right, that are of degree 3 
and 4. We have $A\left(G_{n}^{\prime}\right)=4$ (verified, e.g., by the 4-cycle $\left.a_{2}, a_{4}, a_{6}, a_{5}\right), A_{c L}\left(G_{n}^{\prime}\right)=n-4$ (take all vertices except $a_{1}, a_{3}$ and the symmetric ones in the left gadget), $A_{L}\left(G_{n}^{\prime}\right) \approx 2 n / 3$ (also here a cycle of size 3, for example $a_{1}, a_{2}, a_{3}$, can be supplemented on the path by pairs of consecutive vertices $\left.a_{6}, a_{7}, \ldots\right)$.

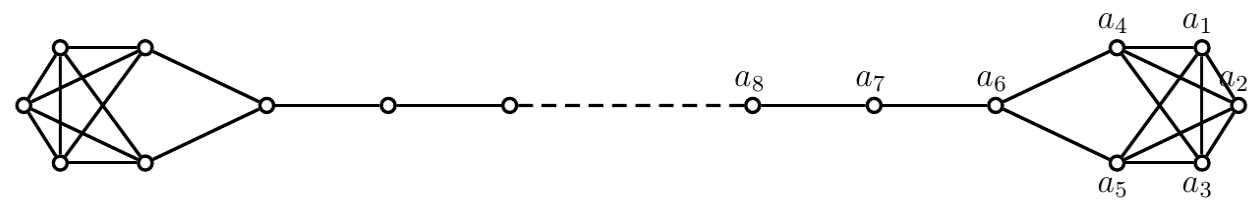

Figure 2: A graph $G_{n}^{\prime}$ with $A\left(G_{n}^{\prime}\right)<A_{L}\left(G_{n}^{\prime}\right)<A_{c L}\left(G_{n}^{\prime}\right)$

Theorem 4 If $G$ is a 3-regular graph then $\hat{A}_{L}(G)>n / 2$, and if $G$ is 3-regular and connected then also $\hat{A}_{c L}(G)>n / 2$. Moreover, a locally minimal strong defensive alliance larger than $n / 2$ can be found in polynomial time for both cases.

Proof. We note first that it suffices to prove the theorem for connected graphs. Indeed, if $G$ is disconnected, with components $G_{1}, \ldots, G_{s}$, then clearly $\hat{A}_{L}(G)=\sum_{i=1}^{s} \hat{A}_{L}\left(G_{i}\right)>$ $\sum_{i=1}^{s}\left|V\left(G_{i}\right)\right| / 2=n / 2$ follows once the connected case is settled.

Hence assume that $G$ is connected. We describe a polynomial-time procedure that generates a locally minimal strong defensive alliance $D_{L}$ and a connected locally minimal strong defensive alliance $D_{c L}$, such that $D_{L} \subseteq D_{c L}$ and $\left|D_{L}\right|>n / 2$. Initially let $D:=V$. Of course, $G$ itself is a connected strong defensive alliance. In the first phase of the algorithm, in each step, search for a vertex $v$ such that $D \backslash\{v\}$ is a strong defensive alliance, moreover the induced subgraph $G[D \backslash\{v\}]$ is connected. If no such $v$ exists, then the first phase terminates and we set $D_{c L}:=D$, otherwise we continue with $D:=D \backslash\{v\}$. The second phase applies essentially the same steps, except that now $v$ can also be a cut vertex of $G[D]$, i.e., from then on the connectivity constraint is dropped. The second phase terminates when $D \backslash\{v\}$ fails to be a strong defensive alliance, for every $v \in D$. We then define $D_{L}:=D$ (where $D_{L}=D_{c L}$ may occur).

It is clear by definition that $D_{L}$ is a locally minimal strong defensive alliance and $D_{c L}$ is a connected locally minimal strong defensive alliance. Since $G$ is 3-regular, after each step the induced subgraph $G[D]$ has minimum degree 2 , therefore when we move a vertex $v$ from $D$ to $V \backslash D$, this $v$ becomes either an isolated vertex or a pendant vertex in the re-defined $G[V \backslash D]$. Consequently, $G\left[V \backslash D_{L}\right]$ is acyclic.

Consider any tree component $T$ of $G\left[V \backslash D_{L}\right]$. Say, $T$ has $t$ vertices. The degree sum in $G[T]$ is $2 t-2$, hence 3-regularity implies that there are exactly $t+2$ edges from $T$ to $D_{L}$, i.e., more edges than $|V(T)|$. Since all degrees inside $G\left[D_{L}\right]$ are at least 2 , the edges from $V \backslash D_{L}$ to $D_{L}$ have mutually distinct endpoints in $D_{L}$. This implies $\left|D_{L}\right|>|V|-\left|D_{L}\right|$, thus

$$
\hat{A}_{c L}(G) \geq\left|D_{c L}\right| \geq\left|D_{L}\right|>n / 2
$$


and of course $\left|D_{L}\right|$ is a lower bound on $\hat{A}_{L}(G)$. It is also clear that the above steps can be performed efficiently.

Consider in the following a simple computational aspect. It is clear that (connected) local minimality of a (strong) defensive alliance can be detected in polynomial time. This is less clear for the (more usual) inclusion-wise notion of minimality, that is, for global minimality. In fact, by its definition, this notion of global minimality seems to require going through all subsets of the alliance in question. However, we can establish the following result.

Proposition 5 There is a polynomial-time algorithm to determine whether a (strong) defensive alliance $D$ is minimal or not.

Proof. We describe first an algorithm to determine if a vertex set $D$ is a minimal defensive alliance. Consider some vertex $v \in D$. If $D \backslash\{v\}$ is a defensive alliance, then we know that $D$ is not minimal and we can stop. If $D \backslash\{v\}$ is not a defensive alliance, then there must be a reason for this. Namely, while $\left|N_{G}[u] \cap D\right| \geq\left|N_{G}(u) \backslash D\right|$ for all $u \in D$, this condition is violated for $D^{\prime}=D \backslash\{v\}$. Hence, there is some vertex $u \in D^{\prime}$ such that $\left|N_{G}[u] \cap D^{\prime}\right|<\left|N_{G}(u) \backslash D^{\prime}\right|$. Clearly, $u \in N_{G}(v)$. Hence, in order to find a subset of $D^{\prime}$ that is a defensive alliance, any $x \in D^{\prime}$ that satisfies $\left|N_{G}[x] \cap D^{\prime}\right|<\left|N_{G}(x) \backslash D^{\prime}\right|$ must be removed from $D^{\prime}$. The set $D^{\prime \prime}$ obtained this way might be a defensive alliance (in which case we can terminate the procedure), or it is empty (which causes us to conclude that the $v \in D$ that we originally considered cannot be removed in order to produce a subset of $D$ that is a defensive alliance), or we find (recursively) more vertices that should be removed. Doing this kind of testing for all $v \in D$ allows us to conclude (in polynomial time) whether or not $D$ is minimal.

The algorithm for determining if a set $D$ is a minimal strong defensive alliance is very similar, we only have to change the condition that each vertex must satisfy.

The preceding result has the following (trivial) consequences; recall that the $O^{*}$-notation neglects polynomial factors.

Corollary 6 There are algorithms that compute $A(G), \hat{A}(G), A_{L}(G), \hat{A}_{L}(G), \hat{A}_{c L}(G)$ and $A_{c L}(G)$ for a given graph of order $n$ in time $O^{*}\left(2^{n}\right)$.

For the following results, it is important to know that Hamiltonian Cycle (i.e., given a graph $G$, is $G$ Hamiltonian?) is NP-hard. Related decision problems are LONGEST CyCle (i.e., given a graph $G$ and an integer $k$, does $G$ possess a cycle on at least $k$ vertices as a subgraph?) and Longest PATH (i.e., given a graph $G$ and an integer $k$, does $G$ possess a path on $k$ vertices as a subgraph?); slightly abusing terminology, we name the corresponding maximization problems the same. Another important NP-hard problem is Minimum Maximal Matching, i.e., given a graph $G$ and an integer $k$, does $G$ possess an inclusion-wise maximal matching with at most $k$ edges? 


\section{Complexity results}

In this section, we show that computing all these six numbers $A(G), A_{L}(G), A_{c L}(G)$ and $\hat{A}(G), \hat{A}_{L}(G), \hat{A}_{c L}(G)$ is NP-hard. Together with Proposition 5 this means that the six decision problems associated to these graph parameters are NP-complete. We also consider these parameters under the perspective of approximability and concerning the impossibility for certain exact algorithms, assuming the Exponential Time Hypothesis (or ETH for short) to hold. ETH basically states that there are no sub-exponential algorithms for solving 3SAT, one of the core problems of NP-completeness theory. Recall that if ETH is true, then $\mathrm{P}$ is not equal to NP, but if ETH fails, then it is still unclear if $\mathrm{P}$ is equal to NP or not. For more details, we refer to [12].

In order to get the NP-hardness in the globally minimal case we use the following remarks. (i) In a cubic graph, finding a globally minimal strong defensive alliance of maximum size is equivalent to finding a longest chordless cycle (or a maximum induced cycle). (ii) In a graph with degrees 3 or 4 , a globally minimal defensive alliance of maximum size corresponds to a longest chordless path between two vertices of degree 3 where vertices inside the path have degree 4 or a longest chordless cycle among vertices of degree 4 .

Theorem 7 Deciding if a graph contains a globally minimal strong defensive alliance of size at least $k$ is NP-complete, even for cubic graphs. Moreover, deciding if a graph contains a globally minimal defensive alliance of size at least $k$ is NP-complete, even for graphs of degree 3 or 4 .

Proof. Both decision problems belong to NP, due to Proposition 5.

In order to obtain the NP-hardness result for the strong version, we establish a polynomial reduction from LONGEST CYCLE on cubic graphs proved NP-hard in [1]. Given a graph $G=(V, E),|V|=n, V=\left\{v_{1}, \ldots, v_{n}\right\},|E|=m=3 n / 2$ and an integer $k$ we construct an instance of our problem $G^{\prime}=\left(V^{\prime}, E^{\prime}\right)$ as follows (see Figure 3): each edge $v_{i} v_{j}$ of $E$ is replaced by the edges $v_{i} a_{i j}, a_{i j} b_{i j}, a_{i j} d_{i j}, b_{i j} c_{i j}, b_{i j} d_{i j}, c_{i j} d_{i j}, c_{i j} v_{j}$ where $a_{i j}, b_{i j}, c_{i j}, d_{i j}$ are new vertices. Thus $G^{\prime}$ contains $n+4 m=7 n$ vertices and $7 m=21 n / 2$ edges. We show that $G$ contains a cycle of size at least $k$ if and only if $G^{\prime}$ contains an induced cycle of size at least $4 k$.

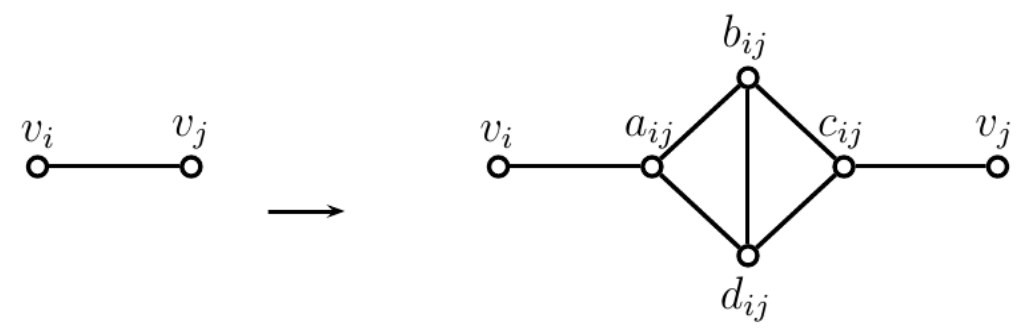

Figure 3: The replacement gadget of an edge $v_{i} v_{j}$ 
Let $C$ be a cycle of size at least $k$ in $G$. Then the cycle $C^{\prime}$ in $G^{\prime}$ obtained by replacing any edge $v_{i} v_{j}$ of $C$ by edges $v_{i} a_{i j}, a_{i j} b_{i j}, b_{i j} c_{i j}, c_{i j} v_{j}$ is a chordless cycle of size $4|C|$ that is at least $4 k$.

Consider now a chordless cycle $C^{\prime}$ in $G^{\prime}$ of size at least $4 k$. Then if edges $v_{i} a_{i j}$ and $c_{i j} v_{j}$ are on $C^{\prime}$ then $C^{\prime}$ contains either $a_{i j} b_{i j}$ and $b_{i j} c_{i j}$ or $a_{i j} d_{i j}$ and $c_{i j} d_{i j}$ since $C^{\prime}$ does not contain chords. The cycle $C$ obtained from $C^{\prime}$ by considering edges $v_{i} v_{j}$ when $v_{i} a_{i j}$ and $c_{i j} v_{j}$ are on $C^{\prime}$ is of size at least $k$.

In order to obtain the NP-hardness result for the globally minimal defensive alliance, we establish a polynomial reduction from LONGEST PATH on cubic graphs proved NP-hard in [1]. Given a graph $G=(V, E),|V|=n, V=\left\{v_{1}, \ldots, v_{n}\right\},|E|=m=3 n / 2$ and an integer $k$ we construct an instance of our problem $G^{\prime \prime}=\left(V^{\prime \prime}, E^{\prime \prime}\right)$ using the gadget $H$ from Figure 4. The gadget $H$ corresponds to the complete graph on 5 vertices $K_{5}$ minus one edge, that is, it contains vertices $s, f, g, h, t$ and edges $s f, s g, s h, f g, g h, f h, t f, t g, t h$, i.e., edge st is missing. Graph $G^{\prime \prime}$ is obtained from $G$ as follows: Each edge $v_{i} v_{j}$ of $E$ is replaced by a copy of $H$ denoted $H_{i j}$, with vertices $f_{i j}, g_{i j}, h_{i j}, s_{i j}$ and $t_{i j}$, and we add edges $v_{i} s_{i j}, t_{i j} v_{j}$. At each vertex $v_{j}$ we attached a copy of $H$ denoted $H_{j}$, with vertices $f_{j}$, $g_{j}, h_{j}, s_{j}$ and $t_{j}$, and we add the edge $t_{j} v_{j}$. Thus $G^{\prime \prime}$ has $6 n+5 m=27 n / 2$ vertices and $10 n+11 m=53 n / 2$ edges. Graph $G^{\prime \prime}$ has only vertices of degree 3 and 4 , and the only vertices of degree 3 are vertices $s_{j}, j=1, \ldots, n$. We show that $G$ contains a path of size at least $k$ if and only if $G^{\prime \prime}$ contains an induced path of size at least $4 k+6$ between 2 vertices of degree 3 and containing only vertices of degree 4 .

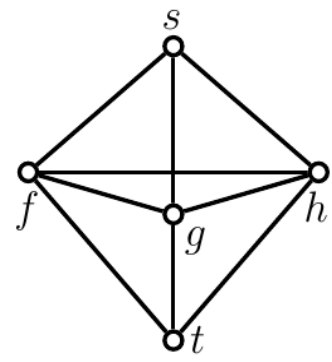

Figure 4: The gadget $H$

Let $P$ be a path of size at least $k$ in $G$ between two vertices $v_{\ell}$ and $v_{p}$. Then the path $P^{\prime \prime}$ in $G^{\prime \prime} s_{\ell} f_{\ell}, f_{\ell} t_{\ell}, t_{\ell} v_{\ell}$, followed by replacing any edge $v_{i} v_{j}$ of $P$ by edges $v_{i} s_{i j}, s_{i j} f_{i j}, f_{i j} t_{i j}, t_{i j} v_{j}$ and finally $v_{p} t_{p}, t_{p} f_{p}, f_{p} s_{p}$ is a chordless path between two vertices of degree 3 and using only vertices of degree 4 inside and of size at least $4 k+6$.

Consider now a chordless path $P^{\prime \prime}$ in $G^{\prime \prime}$ of size at least $4 k+6$ between 2 vertices of degree $3, s_{\ell}$ and $s_{p}$, and containing only vertices of degree 4 . Then $P^{\prime \prime}$ induces a path in $G$ of size at least $k$ between $v_{\ell}$ and $v_{p}$.

Theorem 8 For any $\varepsilon>0$, finding a globally minimal strong defensive alliance of maximum size is not $2^{O\left(\log ^{1-\varepsilon} n\right)}$-approximable on graphs with $n$ vertices, unless $N P \subseteq D T I M E\left(2^{O\left(\log ^{1 / \varepsilon} n\right)}\right)$, 
even for cubic graphs. Moreover, for any $\varepsilon>0$, finding a globally minimal defensive alliance of maximum size is not $2^{O\left(\log ^{1-\varepsilon} n\right)}$-approximable on graphs with $n$ vertices, unless $N P \subseteq D T I M E\left(2^{O\left(\log ^{1 / \varepsilon} n\right)}\right)$, even for graphs of degree 3 or 4 .

Proof. For any $\varepsilon>0$, Longest Path and Longest CyCle on cubic graphs are not $2^{O\left(\log ^{1-\varepsilon} n\right)}$-approximable, unless NP $\subseteq \operatorname{DTIME}\left(2^{O\left(\log ^{1 / \varepsilon} n\right)}\right)$, as shown in [1]. The reductions from the previous proof of Theorem 7 are E-reductions (see [10]) and hence preserve nonapproximability.

It was mentioned in [12] that Hamiltonian CyCle (and hence Longest Cycle) admits no $O^{*}\left(2^{o(n)}\right)$ algorithm under ETH because the standard reduction from 3-SAT is strong. The reduction for HAMILTONIAN CYCLE on cubic (planar) graphs presented in [8] (from 3-SAT) yields a graph whose number of vertices is in a linear relation to the number of variables and clauses of the given 3-SAT instance, so that also such a restricted variant of Hamiltonian CyCle (and hence Longest CyCle) admits no $O^{*}\left(2^{o(n)}\right)$ algorithm under ETH. Re-using our previous construction, we can hence conclude:

Corollary 9 Assuming ETH, there is no $O^{*}\left(2^{o(|V|+|E|)}\right)$-algorithm that decides, given a cubic graph $G=(V, E)$ and some integer $k$, if $G$ contains a globally minimal strong defensive alliance of size at least $k$. Moreover, deciding if a graph contains a globally minimal defensive alliance of size at least $k$ is not possible in time $O^{*}\left(2^{o(|V|+|E|)}\right)$ either, when restricted to graphs with all vertex degrees 3 or 4 , assuming that ETH holds true.

In order to prove NP-hardness for the locally minimal case, we apply a reduction from Minimum Maximal Matching. This problem is well-known to be NP-complete on general graphs. It was proved to be NP-hard even in several special classes of graphs, including planar cubic graphs by Horton and Kilakos [11], and $k$-regular bipartite graphs for any fixed $k \geq 3$ by Demange and Ekim [6]. We have put all these NP-hardness results into one theorem, as the proofs are similar and somehow connected to each other.

Theorem 10 The following problems are NP-complete:

(i) deciding if a graph contains a locally minimal strong defensive alliance of size at least $k$, even for bipartite graphs with average degree less than 3.6;

(ii) deciding if a graph contains a locally minimal defensive alliance of size at least $k$, even for bipartite graphs with average degree less than 5.6;

(iii) deciding if a graph contains a connected locally minimal strong defensive alliance of size at least $k$, even for bipartite graphs with average degree less than $2+\varepsilon$, for any $\varepsilon>0$;

(iv) deciding if a graph contains a connected locally minimal defensive alliance of size at least $k$, even for bipartite graphs with average degree less than $2+\varepsilon$, for any $\varepsilon>0$. 
Proof. All these decision problems are trivially in NP. For NP-hardness we first describe the reductions and proofs for the parts $(i)$ and $(i i)$, which will provide the basis for the other two parts. The next two arguments work for all $n \geq 4$, while the other two will work for $n \geq n_{0}$ for some $n_{0} \leq 10$. Regarding complexity, the small inputs are irrelevant.

(i) For the strong version, we establish a polynomial reduction from Minimum MAXIMAL Matching in 3-regular graphs. Given a 3-regular graph $G=(V, E),|V|=n$, $|E|=m=3 n / 2$, and an integer $k$, we construct an instance of our alliance problem by considering the incidence graph of $G$. It has vertex set $V \cup E$, and there is an edge between $v \in V$ and $e \in E$ if $v$ is an endpoint of $e$. We obtain the graph $G^{\prime}=\left(V^{\prime}, E^{\prime}\right)$ by inserting a new vertex $x$ that is adjacent to every $e \in E$. Since the number of vertices of $G^{\prime}$ is $5 n / 2+1$ and the number of edges is $9 n / 2$, the average degree of $G^{\prime}$ is less than $18 / 5$. We show that $G$ contains a maximal matching of size at most $k$ if and only if $G^{\prime}$ contains a locally minimal strong defensive alliance of size at least $n+m-k$.

If $M$ is a maximal matching in $G$ of size $k$ then $D=V \cup(E \backslash M)$ is a locally minimal strong defensive alliance of $G^{\prime}$. Indeed, every vertex in $V$ has degree at least 2 in $D$ and every vertex in $E$ has degree 2 in $D$. Since $M$ is maximal, it is not possible to remove a vertex from $D \cap E$ and keep a strong defensive alliance. Also, it is not possible to remove a vertex from $D \cap V$ since otherwise some vertices from $D \cap E$ will have degree less than 2 inside $D$.

Consider now a locally minimal strong defensive alliance $D$ in $G^{\prime}$. Any vertex $v \in D$ satisfies the following conditions:

- $d_{D}(v) \geq m / 2$ if $v=x$, and $d_{D}(v) \geq 2$ if $v \in V \cup E$.

Suppose that $D$ has size at least $n+m-k$. We show in the following that there exists a locally minimal strong defensive alliance $D^{\prime}$ in $G^{\prime}$ such that $\left|D^{\prime}\right| \geq|D|$ and $x \notin D^{\prime}$. If $x \notin D$ then $D^{\prime}=D$. If $x \in D$ then the set $D^{\prime}$ can be obtained from $D$ in several steps: remove $x$; add all vertices $u \in V \backslash D$; add a minimal set $A$ of vertices $e \in E \backslash D$ in order that the previously added vertices from $V \backslash(V \cap D)$ satisfy the condition of strong defensive alliance; remove a set $B$ of vertices from $E \cap D$ in order that the new set is a locally minimal strong defensive alliance.

We show now that $\left|D^{\prime}\right| \geq|D|$. Indeed, if $x \in D$, since $D$ is locally minimal, there is at least one vertex $u \in V \backslash D$ and an edge $e \in E \cap D$ such that $e$ is adjacent to $u$ and $x$ in $G^{\prime}$, so $x$ is compensated with the vertices from $\left(V \cap D^{\prime}\right) \backslash D$. Further, every vertex from $A$ has either one or two neighbors in $V \backslash(V \cap D)$, and since every such vertex is of degree 2 in $G^{\prime}[V \cup E]$, we have that every vertex from $A$ has degree 0 or 1 in $V \cap D$, that is, $|N(A) \cap(V \cap D)| \leq|A|$. Finally, for each vertex in $N(A) \cap(V \cap D)$ we removed at most one vertex in $E \cap D$ since each such vertex has to have degree at least 2 in $D^{\prime}$. Thus the number of vertices removed from $E \cap D$ is at most $|A|$.

From $D^{\prime}$ we define $M$ as the set of edges of $G$ that are in $E$ and not in $D^{\prime}$. Since $x \notin D^{\prime}, M$ is a matching; and $M$ is maximal because $D^{\prime}$ is minimal. 
(ii) For locally minimal defensive alliances, we consider a similar reduction except that instead of one vertex $x$ we add two vertices $x_{1}, x_{2}$, joined to every $e \in E$, and moreover, we add one vertex $y$, joined to every $v \in V$. Denote by $G^{\prime \prime}$ the obtained bipartite graph. Since the number of vertices of $G^{\prime \prime}$ is $5 n / 2+3$ and the number of edges is $7 n$, the average degree of $G^{\prime \prime}$ is less than $28 / 5$. We show that $G$ contains a maximal matching of size at most $k$ if and only if $G^{\prime \prime}$ contains a locally minimal defensive alliance of size at least $n+m-k$. We note that $k \leq n / 2$ and $m=3 n / 2$, therefore we have $n+m-k \geq 2 n$.

In one direction, it can be justified as in the previous proof that if $M$ is a maximal matching in $G$, say of size $k$, then $D=V \cup(E \backslash M)$ is a locally minimal defensive alliance of size $n+m-k$ in $G^{\prime \prime}$.

In the other direction, consider now a locally minimal defensive alliance $D$ in $G^{\prime \prime}$. Each vertex $v \in D$ satisfies the following conditions:

- $d_{D}(v) \geq \frac{m-1}{2}$ if $v=x_{1}$ or $v=x_{2}, d_{D}(v) \geq 2$ if $v \in V \cup E$, and $d_{D}(v) \geq \frac{n-1}{2}$ if $v=y$. In particular, if $y \in D$ and $v \in V \cap D$, then the requirement is $d_{V \cup E}(v) \geq 1$.

Suppose that $|D|=n+m-k$. We show in the following that there exists a set $D^{\prime \prime}$ with $\left|D^{\prime \prime}\right| \geq|D|$ such that $D^{\prime \prime}$ is a locally minimal defensive alliance and $x_{1}, x_{2}, y \notin$ $D^{\prime \prime}$. This is very easy if $|D|=2 n$, because every matching in $G$ has at most $n / 2$ edges, hence any maximal one provides a suitable solution and can be determined in polynomial time. For this reason we may and will assume without loss of generality that $|D|>2 n$. The case of $y \notin D$ falls into two simple subcases:

- If $y \notin D$ and at most one of $x_{1}, x_{2}$ is in $D$ then we define $D^{\prime \prime}$ as described above for the strong case, and the proof is done by the argument given in $(i)$.

- If $y \notin D$ and $x_{1}, x_{2} \in D$, then $V \cap D=\emptyset$ since if there is a $v \in V \cap D$ then $v$ can be removed and the remaining set is also a defensive alliance. Consequently, $|D|=\left\lfloor\frac{m}{2}\right\rfloor+2=\left\lfloor\frac{3}{4} n\right\rfloor+2<2 n$, so that this case is excluded.

It remains to study the case $y \in D$. We are going to prove that this assumption implies $|D| \leq 2 n$, thus it cannot occur under the condition $|D|>2 n$.

Let us introduce the notations $V^{\prime}=D \cap V, E^{\prime}=D \cap E, n^{\prime}=\left|V^{\prime}\right|$, and $m^{\prime}=\left|E^{\prime}\right|$. Also, let $d^{\prime}(x)$ denote the degree of an $x \in D$ in the subgraph induced by $D$ in $G^{\prime \prime}$. The minimality of $D$ means that each $x \in D$ has at least one neighbor $x^{\prime} \in D$ such that $d^{\prime}\left(x^{\prime}\right)=\left\lfloor\frac{1}{2} d_{G^{\prime \prime}}\left(x^{\prime}\right)\right\rfloor$.

Next, we prove that $m^{\prime} \leq\left\lfloor\frac{3}{4} n\right\rfloor$. This is immediate (in fact with equality) if each neighbor $v$ of some $e \in E^{\prime}$ has $d^{\prime}(v) \geq 3$, because only $x_{1}$ or $x_{2}$ (or both) can play the role of a neighbor $x^{\prime}$ of $v$ whose $D$-degree is $\left\lfloor\frac{1}{2} d_{G^{\prime \prime}}\left(x^{\prime}\right)\right\rfloor$. If $m^{\prime}$ is larger, then for each $e \in E^{\prime}$ we can specify a $v_{e} \in V^{\prime}$ whose unique neighbor in $E^{\prime}$ is $e$. Let $V^{\prime \prime}$ denote the set of those $v_{e}$; we have $\left|V^{\prime \prime}\right|=m^{\prime}$. Each $e \in E^{\prime}$ has its other neighbor in $V \backslash V^{\prime \prime}$, 
hence there are exactly $m^{\prime}$ edges joining $E^{\prime}$ with $V \backslash V^{\prime \prime}$. On the other hand, there exist at most $3\left(n-m^{\prime}\right)$ such edges, since $G$ is 3 -regular. This implies the claimed inequality $m^{\prime} \leq\left\lfloor\frac{3}{4} n\right\rfloor$.

Now we are in a position to prove that $y \in D$ implies $|D| \leq 2 n$. Note that $n \geq 4$ and $n$ is even. If $n^{\prime}=\frac{1}{2} n$ (the smallest possible case, as $d_{G^{\prime \prime}}(y)=n$ ) then

$$
|D| \leq n^{\prime}+m^{\prime}+3 \leq \frac{1}{2} n+\left\lfloor\frac{3}{4} n\right\rfloor+3 \leq 2 n
$$

is valid for all even integers $n \geq 4$. Also, if $n^{\prime}$ is in the range $\frac{1}{2} n+1 \leq n^{\prime} \leq n-1$, we cannot have both $x_{1} \in D$ and $x_{2} \in D$ because otherwise $D \backslash\{v\}$ would be a defensive alliance for any $v \in V^{\prime}$, contradicting the minimality of $D$. Thus, in this case,

$$
|D| \leq n^{\prime}+m^{\prime}+2 \leq n-1+\left\lfloor\frac{3}{4} n\right\rfloor+2 \leq 2 n
$$

as $\left\lfloor\frac{3}{4} n\right\rfloor \leq n-1$ holds for all even $n \geq 4$. Finally, if $n^{\prime}=n$, then every $e \in E^{\prime}$ has both of its neighbors $v$ in $V^{\prime}$, therefore the presence of $x_{1}$ or $x_{2}$ in $D$ would imply the contradiction that $D \backslash\{v\}$ is a defensive alliance for any $v$. This implies

$$
|D| \leq n^{\prime}+m^{\prime}+1 \leq n+\left\lfloor\frac{3}{4} n\right\rfloor+1 \leq 2 n
$$

which is the same conclusion as the one for $n^{\prime}<n$.

This contradiction completes the proof of part $(i i)$.

Now we turn to the parts $(i i i)$ and $(i v)$, assuming that $n$ is sufficiently large. We shall make use of the graphs $G^{\prime}$ and $G^{\prime \prime}$ constructed in $(i)$ and (ii), respectively. The substantial difference between $(i)-(i i)$ and $(i i i)-(i v)$ is that the cut vertices do not have to satisfy any degree constraints in a locally minimal alliance. For this reason we first describe both constructions and prove that if some cut vertices of an alliance $D$ arise from the vertices of $G^{\prime}$ or $G^{\prime \prime}$, then $D$ cannot be too large. Afterwards we complete the proofs for $($ iii) and (iv) separately. Note that here we do not analyze small graphs anymore, we assume that $n$ is sufficiently large.

From any 3-regular graph $G=(V, E)$, the graphs $G_{c}^{\prime}$ for (iii) and $G_{c}^{\prime \prime}$ for $(i v)$ are constructed as follows. Both constructions share the idea to take two slightly modified copies of a previously constructed graph and join them by a path of sufficient length in order to arrive at the desired average degree upper bound. We refrain from giving an illustration, as similar ideas were used in the introductory examples.

- Supplement $G^{\prime}$ with two new vertices $y_{1}, y_{2}$ joined completely to the set $V$, i.e., $G^{\prime}\left[V \cup\left\{y_{1}, y_{2}\right\}\right]$ is a complete bipartite graph $K_{2,|V|}$; join a new vertex $z$ to both $y_{1}$ and $y_{2}$; take two vertex-disjoint copies of this graph, and connect them with a path $P$ whose endpoints are the copies of $z$ and whose length is $C n$, where $C$ is a sufficiently large constant. This yields an instance $G_{c}^{\prime}$ for the connected strong defensive alliance problem. Since the average degree of $G^{\prime}$ is less than $18 / 5$, there is a suitable choice of $C$ to ensure an average degree of at most $2+\varepsilon$ in $G_{c}^{\prime}$. 
- The construction of the graph $G_{c}^{\prime \prime}$ is fairly similar, now starting from $G^{\prime \prime}$, in which we rename $y$ as $y_{1}$. Supplement $G^{\prime \prime}$ with two new vertices $y_{2}, y_{3}$ joined completely to the set $V$; join a new vertex $z$ to all of $y_{1}, y_{2}, y_{3}$; take two vertex-disjoint copies of this graph, and connect them with a path $P$ whose endpoints are the copies of $z$ and whose length is $C n$, where $C$ is a sufficiently large constant. This yields an instance $G_{c}^{\prime \prime}$ for the connected defensive alliance problem. Here again, there is a suitable choice of $C$ to ensure an average degree of at most $2+\varepsilon$ in $G_{c}^{\prime \prime}$.

To unify notation, we rename $x$ of $G^{\prime}$ as $x_{1}$. Throughout, $D$ will denote a connected defensive alliance or a connected strong defensive alliance, locally minimal in either case. From above, we keep the notation $n^{\prime}=|V \cap D|$ and $m^{\prime}=|E \cap D|$, where $V$ and $E$ are meant as the corresponding sets in a copy of $G^{\prime}$ or $G^{\prime \prime}$ in the construction. The next part of the discussion assumes that $y_{1} \in D$ at an end of $P$ - more precisely that the corresponding copy of $y_{1}$ belongs to $D$; this will later turn out to be necessary in order to have a large $D$ - and analyzes the possibilities of cut vertices in the subgraph induced by $D$ in $G_{c}^{\prime}$ or $G_{c}^{\prime \prime}$.

Fact $\mathbf{X}$. The vertex $x_{1}$ cannot be a cut vertex in $D$.

Indeed, otherwise there would be two edges $e, e^{\prime} \in E$ which are in distinct components of $D \backslash\left\{x_{1}\right\}$. Only one of $e$ and $e^{\prime}$ - say, $e$ - can be adjacent to $V \cap D$, because $V \subset N\left(y_{1}\right)$. By the alliance degree condition this requires the two neighbors $x_{1}, x_{2}$ for $e^{\prime}$ to lie inside of $D$, but then $x_{1}$ cannot be a cut vertex.

Fact E. If some $e \in E$ is a cut vertex in $D$ and $n$ is sufficiently large, then $|D|$ has fewer than $2 n$ vertices in $G^{\prime} \cup\left\{y_{1}, y_{2}\right\}$ or in $G^{\prime \prime} \cup\left\{y_{1}, y_{2}, y_{3}\right\}$.

To prove this, we first note that $e$ cannot separate vertices of $V \cap D$ from each other, because $V \subset N\left(y_{1}\right)$. Hence $e$ separates $x_{1}$ or $x_{2}$ (or both) from $V \cap D$. In particular, we may assume that $x_{1} \in D$, and this implies $m^{\prime} \geq \frac{m-1}{2}>1$. Thus there exists $e^{\prime} \in E \cap D$ with $e^{\prime} \neq e$ and $\left(N\left(e^{\prime}\right) \cap V\right) \cap D=\emptyset$. Such an $e^{\prime}$ needs two neighbors in $D$, which can now only be $x_{1}$ and $x_{2}$. This is impossible in $G_{c}^{\prime}$. In $G_{c}^{\prime \prime}$ assume that $x_{1}, x_{2} \in D$. Since $e^{\prime}$ has no other neighbors, and $D \backslash\left\{e^{\prime}\right\}$ is not an alliance, we obtain that $e^{\prime}$ is locally critical for $x_{1}$, therefore $E \cap D$ contains exactly $\left\lceil\frac{m-3}{2}\right\rceil$ vertices different from $e$. From them there are at least $m-3$ edges to $V$, incident with at least $\frac{m-3}{3}=\frac{n}{2}-1$ vertices of $V$, none of which can belong to $D$. Consequently $n^{\prime}+m^{\prime}<2 n-5$ if $n$ is sufficiently large, thus $D$ cannot have $2 n$ vertices or more in this part of the graph even if we count all of $x_{1}, x_{2}, y_{1}, y_{2}, y_{3}$.

Fact V. If some $v \in V$ is a cut vertex in $D$ and $n$ is sufficiently large, then $|D|$ has fewer than $2 n$ vertices in $G^{\prime} \cup\left\{y_{1}, y_{2}\right\}$ or in $G^{\prime \prime} \cup\left\{y_{1}, y_{2}, y_{3}\right\}$.

Choose an $e \in E \cap D$ which becomes separated from $y_{1}$ in the subgraph $D \backslash\{v\}$. We may assume that $e$ is not a cut vertex, otherwise Fact $E$ applies and the proof is done. The unique neighbor of this $e$ in $V \cap D$ is $v$, therefore $e$ needs a further neighbor in $D$; hence $x_{1} \in D$ (or $x_{2} \in D$ ). Moreover, $v$ has a further neighbor $e^{\prime} \in E \cap D$ because $e$ is not a cut vertex. Hence $v$ has at least three neighbors in $D$, but $D \backslash\{e\}$ is not an alliance while still connected, thus $m^{\prime}=\left\lceil\frac{m-1}{2}\right\rceil$ or $m^{\prime}=\left\lceil\frac{m}{2}\right\rceil$. Among those $m^{\prime}$ vertices at least $m^{\prime}-3$ (namely the non-neighbors of $v$ ) have no neighbors in $V \cap D$. From each of them, two 
edges go to $V$, hence $n-n^{\prime}=|V \backslash D| \geq 2 m^{\prime} / 3-c \approx n / 2-c$ for a small constant $c$. This leads to the same conclusion as above, namely $n^{\prime}+m^{\prime}<2 n-5$ if $n$ is sufficiently large.

The relevance of the upper bound in Facts E and V is that - as we shall see soon such a small $D$ cannot be an optimal solution to the alliance problems considered. Hence, in the rest of the proof we restrict our attention to alliances in which no vertex originating from $G^{\prime}$ or $G^{\prime \prime}$ is a cut-vertex, except for $y_{1}$.

(iii) For connected strong defensive alliances we consider the graph $G_{c}^{\prime}$.

A strong defensive alliance $D$ puts the following set of conditions for a vertex $v \in D$; for simplicity we omit the word "copy" from phrasing, e.g., ' $v=x$ ' will mean that $v$ is one of the two copies of $x$ in $G_{c}^{\prime}$.

- $d_{D}(v) \geq \frac{1}{2} m$ if $v=x, d_{D}(v) \geq 2$ if $v \in E, d_{D}(v) \geq 3$ if $v \in V, d_{D}(v) \geq \frac{1}{2}(n+1)$ if $v=y_{j}, d_{D}(v) \geq 2$ if $v=z$, and $d_{D}(v) \geq 1$ if $v$ is an internal vertex of $P$.

We claim that a largest connected locally minimal strong defensive alliance of $G_{c}^{\prime}$ can be obtained by taking a largest locally minimal strong defensive alliance in each of the two copies of $G^{\prime}$, plus exactly one $y_{j}(j=1$ or $j=2)$ in each copy, plus the path $P$ connecting the two copies. (This means, in particular, that the two copies of $V$ can entirely be contained in the alliance in question.) It is clear that such a subgraph satisfies the degree conditions of a strong alliance, and it is minimal because the vertices in $P$ and the $y_{j}$ are critical for connectivity, and the vertices in the copies of $V$ and $E$ cannot be deleted due to the degree constraints for $E$ and $V$, respectively. Consider any connected locally minimal strong defensive alliance $D$ in $G_{c}^{\prime}$. If no $y_{j}$ is involved in $D$ at some end of $P$, then its neighbor $z$ cannot belong to $D$, and then the internal vertices of $P$ but the one preceding the other copy of $z$ would be removable (unless $D$ is an internal edge of $P$ ), hence at most 4 vertices of $D$ are outside a copy of $G^{\prime}$. Thus, in this case we have $|D| \leq 5 n / 2+5$, while the alliance constructed above has at least $4 n+|P|$ vertices.

Hence, we may assume without loss of generality that $D$ contains one or two of the $y_{j}$ in each copy. Consider now the situation in any one copy. If $D$ contains precisely one $y_{j}$, then this $y_{j}$ is critical for connectivity, and its presence reduces the degree constraints within the corresponding copy of $G^{\prime}$ as follows:

- $d_{D}(v) \geq m / 2$ if $v=x$, and $d_{D}(v) \geq 2$ if $v \in V \cup E$.

This is exactly the set of conditions listed in $(i)$, consequently in this case the maximum of $|D|$ is attained by precisely the construction described above.

Suppose now that $D$ contains both $y_{1}$ and $y_{2}$ in the copy considered. Then the degree constraints within the corresponding copy of $G^{\prime}$ are modified as follows:

- $d_{D}(v) \geq m / 2$ if $v=x, d_{D}(v) \geq 2$ if $v \in E$, and $d_{D}(v) \geq 1$ if $v \in V$. 
This situation has also been analyzed already, namely in part $(i i)$, within the subcase $y \in D$ (this corresponds to the sub-subcase $x_{1} \in D, x_{2} \notin D$ ), where we have seen that it cannot lead to any alliance larger than the one constructed above.

(iv) For connected (not strong) defensive alliances we consider the graph $G_{c}^{\prime \prime}$.

A defensive alliance $D$ now puts the following set of conditions for a vertex $v \in D$; as in (iii), also here we omit the word "copy" from phrasing.

- $d_{D}(v) \geq \frac{m-1}{2}$ if $v=x_{i}, d_{D}(v) \geq 2$ if $v \in E, d_{D}(v) \geq 3$ if $v \in V, d_{D}(v) \geq n / 2$ if $v=y_{j}$, and $d_{D}(v) \geq 1$ if $v$ is on $P$ (also including its end $z$ ).

Now a largest connected locally minimal defensive alliance of $G_{c}^{\prime \prime}$ can be obtained by taking a largest locally minimal defensive alliance in each of the two copies of $G^{\prime}$, plus exactly one $y_{j}(j \in\{1,2,3\})$ in each copy, plus the path $P$ connecting the two copies. It can be seen as before that this set satisfies the requirements.

Consider any connected locally minimal defensive alliance $D$ in $G_{c}^{\prime \prime}$. We see that $D$ contains at least one $y_{j}$ from each copy of $G^{\prime \prime}$, for otherwise $|D|$ is far from being largest. Hence, we may assume without loss of generality that $D$ contains one or two or three of the $y_{j}$ in each copy. Consider now the situation in any one copy. If $D$ contains precisely one $y_{j}$, then this $y_{j}$ is critical for connectivity, and its presence reduces the degree constraints inside the corresponding copy of $G^{\prime}$ as follows:

- $d_{D}(v) \geq \frac{m-1}{2}$ if $v=x_{i}$, and $d_{D}(v) \geq 2$ if $v \in V \cup E$.

Compared to the subcase $y \notin D$ of $(i i)$ the only difference is that $y_{j}$ now requires $n / 2-1$ vertices, one fewer than previously. However, this relaxed condition has no essential effect on the argument given earlier. Indeed, with reference to the relevant paragraph of the proof of $(i i)$, if $D$ contains at most one of $x_{1}$ and $x_{2}$ then the proof goes back to a subcase of part $(i)$, where no $y$ occurs (hence the actual degree of $y_{j}$ is irrelevant); and if both $x_{1}$ and $x_{2}$ are in $D$ then the difference is that instead of $V \cap D=\emptyset$ we now must have $|V \cap D|=n / 2-1$, hence we obtain the upper bound $|D| \leq 3 n / 4+n / 2+c$ with a small constant $c$, which is still smaller than $2 n$ if $n$ is sufficiently large.

If $D$ contains two of the vertices $y_{1}, y_{2}, y_{3}$ in the copy of $G^{\prime \prime}$, then the degree constraints inside the corresponding copy of $G^{\prime}$ are modified to

- $d_{D}(v) \geq \frac{m-1}{2}$ if $v=x_{i}, d_{D}(v) \geq 2$ if $v \in E$, and $d_{D}(v) \geq 1$ if $v \in V$.

This is essentially the case $y \in D$ of $(i i)$. We note that the $y_{j}$ cannot be critical for connectivity anymore, therefore the degree requirements concerning a critical neighbor are valid also here for the vertices. The corresponding computation in $(i i)$ yields an upper bound around $7 n / 4$.

Finally, if all of $y_{1}, y_{2}, y_{3}$ are in $D$, then we have the degree requirements 
- $d_{D}(v) \geq \frac{m-1}{2}$ if $v=x_{i}, d_{D}(v) \geq 2$ if $v \in E$, and no condition if $v \in V$.

Moreover, $y_{1} \in D$ requires $n^{\prime} \geq n / 2$. By assumption, the set $D \backslash\left\{y_{1}\right\}$ is not an alliance, therefore $V \cap D$ contains a vertex $v$ whose only neighbors in $D$ are $y_{1}, y_{2}, y_{3}$. Since $D \backslash\{v\}$ is not an alliance either, we see that $n^{\prime}=n / 2$. Further, the removal of any $e \in E \cap D$ violates the alliance property, which can happen only to $x_{1}$ or $x_{2}$, thus $m^{\prime}=\left\lceil\frac{m-1}{2}\right\rceil$. In this way we again obtain an upper bound around $7 n / 4$.

This completes the proof of the theorem.

Proposition 11 Unless ETH fails, there is no algorithm that determines if there is a locally minimal (strong) defensive alliance of size at least $k$ in a given graph $G$ of order $n$ in time $O\left(2^{o(n)}\right)$, even on bipartite graphs with the restrictions from the preceding theorem. A similar statement holds for the connected locally minimal (strong) defensive alliance problems.

Proof. It has been argued in [16] that no $O\left(2^{o(n)}\right)$ algorithm exists for solving VERTEX Cover on cubic graphs unless ETH fails. Consider now the reduction of Theorem 1 in [19]. This shows that no $O\left(2^{o(n)}\right)$ algorithm exists for solving Edge Dominating SeT on subcubic bipartite graphs unless ETH fails, which is equivalent to the non-existence of an $O\left(2^{o(n)}\right)$ algorithm for Minimum Maximal Matching in subcubic bipartite graphs of order $n$. Zito has shown in [21, Lemma 29] how to replace vertices of degree one by four-vertex-graphs, so that an ETH-based lower bound also holds for Minimum MAXIMAL Matching in bipartite graphs with vertex degrees two or three. The construction of Theorem 7 in [6] shows that there is no $O\left(2^{o(n)}\right)$ algorithm for solving Minimum MaXimaL MATCHING on cubic bipartite graphs, unless ETH fails. The reasoning of the preceding theorem shows the claim. For the connected locally minimal (strong) defensive alliance problems, observe that the resulting graphs have a linear number of vertices (compared to the original graph as an instance of Minimum Maximal Matching); notice that the linearity factor depends on the chosen $\varepsilon$.

The preceding proposition shows that the algorithms mentioned in Corollary 6 for determining $A_{L}(G)$ and $\hat{A}_{L}(G)$, as well as the connected variants, are essentially optimal.

Concerning the inapproximability of our problem, we remark that the reductions in Theorem 10 are L-reductions (see [10]). Using the result from [5] that Minimum MaximaL MATCHING in cubic graphs is NP-hard to approximate within a factor $1+\frac{1}{487}$, we can conclude that the optimization versions of all the problems studied in Theorem 10 have no polynomial-time approximation scheme (that is, they do not admit a polynomial-time $(1+\epsilon)$-approximation algorithm for very small $\epsilon>0)$ if $\mathrm{P} \neq \mathrm{NP}$.

\section{Conclusions}

In this paper, we commenced a complexity-theoretic study of several variations of maximum minimal defensive alliances. Many graph-theoretic questions are still to be explored for the new parameters that we introduced. 
Also, we think that the connectivity requirement that we introduced for the locally minimal type are of more general interest for any type of alliance problem, because (in particular for the strategic motivations for these graph parameters) it seems reasonable to look for connected alliances, as this also models the aspect of mutual (quick) help.

Finally, notice that the different notions of minimality can also be studied in connection with other types of alliances with the same motivation. This is also left for future work.

\section{Acknowledgements}

We are grateful to the referee for her/his thorough reading and many comments that have made the paper better readable.

\section{References}

[1] C. Bazgan, M. Santha, and Zs. Tuza. On the approximation of finding a(nother) Hamiltonian cycle in cubic Hamiltonian graphs. Journal of Algorithms, 31(1):249268, 1999.

[2] C. Bazgan, Zs. Tuza, and D. Vanderpooten. On the Existence and Determination of Satisfactory Partitions in a Graph. Proceedings of the 14th International Symposium on Algorithms and Computation, ISAAC, volume 2906 of LNCS, 444-453. Springer 2003.

[3] C. Bazgan, Zs. Tuza, and D. Vanderpooten. Satisfactory graph partition, variants, and generalizations. European Journal of Operational Research, 206(2):271-280, 2010.

[4] R. Carvajal, M. Matamala, I. Rapaport, and N. Schabanel. Small alliances in graphs. In Proceedings of the 32nd International Symposium on Mathematical Foundations of Computer Science, MFCS, volume 4708 of LNCS, pages 218-227. Springer, 2007.

[5] M. Chlebík and J. Chlebíková. Approximation hardness of edge dominating set problems. Journal of Combinatorial Optimization, 11:279-290, 2006.

[6] M. Demange, T. Ekim, and C. Tanasescu. Hardness and approximation of minimum maximal matchings. International Journal of Computer Mathematics, 91(8):16351654, 2014.

[7] G. Flake, S. Lawrence, and C. Lee Giles. Efficient identification of web communities. In Proceedings of the 6th ACM SIGKDD International Conference on Knowledge Discovery and Data Mining, pages 150-160, 2000.

[8] M. R. Garey, D. S. Johnson, and R. E. Tarjan. The planar Hamiltonian circuit problem is NP-complete. SIAM Journal on Computing, 5(4):704-714, 1976. 
[9] A. Grady, F. Knoll, R. Laskar, and D. J. Lipman. Cycle domination, independence and irredundance in graphs. Technical Report arXiv.org:1505.02268, Cornell University, 2015.

[10] D. Hochbaum. Approximation Algorithms for NP-hard Problems. PWS Publishing Co., 1997.

[11] J. D. Horton and K. Kilakos. Minimum edge dominating sets. SIAM Journal of Discrete Mathematics, 6(3):375-387, 1993.

[12] R. Impagliazzo, R. Paturi, and F. Zane. Which problems have strongly exponential complexity? Journal of Computer and System Sciences, 63(4):512-530, 2001.

[13] P. Kristiansen, S. M. Hedetniemi, and S. T. Hedetniemi. Alliances in graphs. Journal of Combinatorial Mathematics and Combinatorial Computing, 48:157-177, 2004.

[14] D. F. Manlove. Minimaximal and maximinimal optimisation problems: a partial orderbased approach. PhD thesis, University of Glasgow, Computing Science, 1998.

[15] J. A. Rodríguez-Velázquez, I. G. Yero, and J. M. Sigarreta. Defensive $k$-alliances in graphs. Applied Mathematics Letters, 22(1):96-100, 2009.

[16] R. B. Sandeep and N. Sivadasan. Parameterized lower bound and improved kernel for diamond-free edge deletion. In T. Husfeldt and I. A. Kanj, editors, 10th International Symposium on Parameterized and Exact Computation, IPEC, volume 43 of LIPIcs, pages 365-376. Schloss Dagstuhl - Leibniz-Zentrum für Informatik, 2015.

[17] K. H. Shafique. Partitioning a graph in alliances and its application to data clustering. Phd thesis, School of Computer Science, University of Central Florida, Orlando, 2004.

[18] J. M. Sigarreta, S. Bermudo, and H. Fernau. On the complement graph and defensive k-alliances. Discrete Applied Mathematics, 157(8):1687-1695, 2009.

[19] M. Yannakakis and F. Gavril. Edge dominating sets in graphs. SIAM Journal of Applied Mathematics, 38(3):364-372, 1980.

[20] I. G. Yero and J. A. Rodríguez-Velázquez. Defensive alliances in graphs: a survey. 2013. https://arxiv.org/pdf/1308.2096v1.pdf. Utilitas Mathematica, to appear.

[21] M. Zito. Randomised techniques in combinatorial algorithmics. PhD thesis, University of Warwick, UK, 1999. 